\title{
Design and Application of Innovative Teaching Platform Based on Fog Computing
}

\author{
Jiejian Cai, Baoling Qin*, Fenglin Zheng, Sina Li, Yunshi Luo, Jianwei Zhang \\ College of Electronic Information Engineering, Foshan University \\ Foshan, China
}

\begin{abstract}
Fog computing is a new type of calculation model in recent years, which shares part of the pressure of cloud computing storage, calculation and processing. Compared with the traditional teaching methods, this paper proposes to use fog computing and big data technology features to build an innovative teaching platform system and the fog model based on fog computing, which can reduce network broadband pressure, reduce the burden on cloud servers, improve comprehensive computing ability, and reduce delay. In addition, it can enhance the exchange of information between teachers and students, guarantee the full range of service levels between teachers and students, and mainly solve the problem of network congestion caused by big data teaching resources. The platform design mainly realizes the functions of resource sharing, teacher-student interaction, effect feedback, intelligent management, etc. Besides, it also can intelligently analyze student behavior, intelligently predict students' favorite courses recommend relevant courses for students to choose, and reform the teaching methods and management modes to improve the interaction between teaching and learning, and improve students' enthusiasm and efficiency in learning.
\end{abstract}

Keywords-Cloud Computing; Fog Computing; Fog Model; Distributed Computing; Innovative Teaching Platform

\section{INTRODUCTION}

With the development of the Internet, the network teaching technology in the era of big data is gradually being embedded in the traditional teaching mode, breaking through the limitations of learners' time and space, and having more options for selecting innovative learning according to their own characteristics, therefore, learners are able to achieve the greatest sharing of educational resources and promote the possibility of "lifelong learning". The current network teaching platform is combining the comprehensive application of technologies such as big data, cloud computing, Internet of Things and fog computing and having been developed rapidly. However, the learning style based on big data technology which emerges as the times require in teaching platform transmits the useless and invalid data to the cloud server, exposing its obvious shortcomings in the process of the data processing, for example, it takes a long time to process data; data is easy to lose; bandwidth resources are insufficient; there is network congestion and other problems. Aiming at the current situation and existing problems of the teaching platform, the design of the architecture which combines innovative teaching platform with fog computing is proposed, and this paper elaborates the role of fog computing in the innovative teaching platform ,how fog computing can provide daily big data information services in the innovative teaching platform and how to achieve distributed computing, how to mitigate storage pressure, how to free up space at any time and other applications, which further promote the development of the online teaching platform, and whose research results have practical application significance in the new teaching mode.

\section{ANALYSIS OF CURRENT STATUS OF TEACHING PLATFORM}

In recent years, under the rapid development of network information technology, the traditional teaching mode is also advancing to the level of network teaching technology. The traditional teaching mode dominated by "blackboard + chalk" is being impacted by a large number of network information teaching technology models ${ }^{[1]}$. This new network teaching mode is not only more novel in form, but also emphasizes the learner centered. The corresponding teaching idea and mode selection is based on learners' personality, thus, the freedom of learning is also relatively wide, and the learners' initiative is fully utilized ${ }^{[2]}$. On the basis of the network teaching platform, the learners' learning content may be updated in real time due to social development and needs, ensuring that the knowledge absorbed by learners can better adapt themselves to personal development in society. The technicalization of the innovative teaching platform network not only expands the existing technology of the education industry, but also further promotes the deeper mining demand for educational resources in the rapid development of society ${ }^{[3]}$. Users in the education industry have relatively high demand for the overall informationization of learning resources, while the old teaching platforms and methods seem inadequate and there exists lots of problems such as insufficient broadband, network congestion, single teaching methods, difficult achieving of the interaction between teachers and students, difficult achieving of students' independent innovation and learning and so on especially with the rapid development of teaching resources based on big data. Therefore, in the process of constructing the data center of the network education platform, it is the hot trend and current status of the innovative teaching platform to explore how to better provide learning users with a more advanced, portable, quickly, high-performance network operation architecture and

2018 Student Academic Fund Project of Foshan University 
infrastructure platform.

\section{Design of InNOVAtive Teaching Platform BASED ON FOG CALCULATION}

\section{A. Key technology applications and features}

\section{1) Cloud computing applications and features}

Cloud computing is a concept put forward by Google engineer Christopher Bihlia. The purpose is to adapt to the rapid development of the network era and make people better deal with the huge data generated by the network infrastructure equipment itself. Although cloud computing can perform real-time, fast and convenient sharing of resources such as network infrastructure devices, internal applications, and stored data by utilizing the Internet and according to users' requirements. However, under the continuous development of a large number of network devices, network services dominated by cloud computing have also experienced obvious problems such as network congestion, slow data processing results, and inaccurate processing of data results. Because the data that is transmitted to the "cloud" is raw data that has not been processed by the IoT device, the data includes a lot of useless data that is not necessary to transmit, which not only occupies limited bandwidth, but also makes cloud withstand the pressure of useless data storage and processing. Besides, it is also prone to security problems such as data loss and leakage and, to a certain extent, it can't meet the low-speed network speed requirements of customers.

\section{2) Fog computing application and characteristics}

In 2012, F. Bonomi et al. proposed the concept of "fog computing" [4]. The idea was mainly to expand and supplement the existing technology of cloud computing, rather than to replace "cloud computing". Fog computing is further extended on the basis of network backbone and data center which are based on cloud computing as the core, and is the "fog server" connected to a large number of infrastructure devices of the Internet of Things. Computing mode has gradually changed from IT service providers' "big cloud" and "social public cloud" advocated by cloud computing to three new computing modes: personal cloud, family cloud and unit cloud. It can be perceived by a huge cloud and big data structure to some extent, and can effectively and quickly extract limited and accurate data content, thereby greatly improving the accuracy and speed of solving problems, realizing more efficient real-time data calculation and storage and reducing the load on cloud computing storage and processing.

Virtual computing, which can also be called edge computing, is a more distributed computing service model based on cloud computing and it is not more complex than cloud computing. It has the characteristics of close proximity to the user, low latency, high confidentiality, high reliability, dense geographical space of the distributed network, multiple nodes, wireless access network equipment, etc, which also makes it have the benefits of high processing efficiency, network environmental security monitoring, real-time analytics, close-range source control capabilities and support for real-time interaction and cloud online analytics. Meanwhile, it support the technical advantages of heterogeneous hardware and software devices in a variety of environmental and other factors. Taking full advantage of the user's most accessible virtualized infrastructure and processing data at the edge of the cloud server greatly reduce the economic cost and provide users with more access to space. Therefore, fog computing is an aggregation of the advantages of cloud computing and at the same time, it improve the deficiencies of cloud computing and extending the server side of cloud computing vacancies. Its application value in the era Internet of Things and Big Data is getting higher and higher, and the application of innovative teaching platform has become a new research hotspot.

\section{3) Big data applications and features}

Since the concept of big data was proposed, the application field has developed rapidly in a few years, and all walks of life are in a hot state. The education industry is no exception. It has developed into a collection of data with high value and many types, also exists in a large variety of structured data, such as pictures, text, audio, video, time, location, and web logs that are found everywhere in innovative teaching platform systems. Because of the large scale of data it integrates, general software tools cannot implement a series of operations such as extracting, processing, and organizing data. It is known from a large number of documents that big data has the characteristics of $5 \mathrm{~V}$, that is, it has a huge amount of data, a fast processing speed, a variety of data types, and the authenticity and effectiveness of data from life. Based on these extensive applications and characteristics, the innovative teaching platform is closely related to big data, so for the calculation and processing of these big data, distributed fog computing solutions must be adopted to make the platform better serve the teaching.

\section{B. Design of Innovative Teaching Platform Based on Fog Computing}

\section{1) Innovative teaching platform architecture}

Different from the traditional teaching methods, the network teaching platform integrates teaching management, teaching curriculum resources, teaching interaction and evaluation, to support online teaching and realize independent, remote and interactive learning ${ }^{[5]}$. Most of the online teaching platforms that are often mentioned today are based on cloud computing to provide support. In the past, the penetration rate of networks and mobile terminals was not high, and the amount of data generated was small, so cloud computing was suitable for users' needs. However, with the rapid development of various science and technology and the continuous popularization of terminals, the data generated is exponentially exploding, and the centralized processing of data information by cloud computing continues to show its drawbacks. It is easy to cause network congestion, too much data load in the cloud and too long delay, which affects the teaching effect. The innovative teaching platform based on fog computing can solve these problems, because the fog computing distributes a large number of fog nodes close to the users, which is equivalent to inserting a layer of fog in the previous cloud computing model to assist cloud computing. The terminal equipment can be directly and quickly accessing 
the nearest fog node for data transmission and processing. Therefore, the transmission efficiency is greatly improved and the network delay is reduced, especially the video transmission delay. At the same time, its characteristic of the distributed computing avoids the massive terminals or users accessing the cloud at the same time, and also solve the problem of network congestion.

The architecture of the innovative teaching platform can be divided into four parts: the Perception Layer, the Network Layer, the Platform Layer and the Application Layer, as shown in Fig.1.

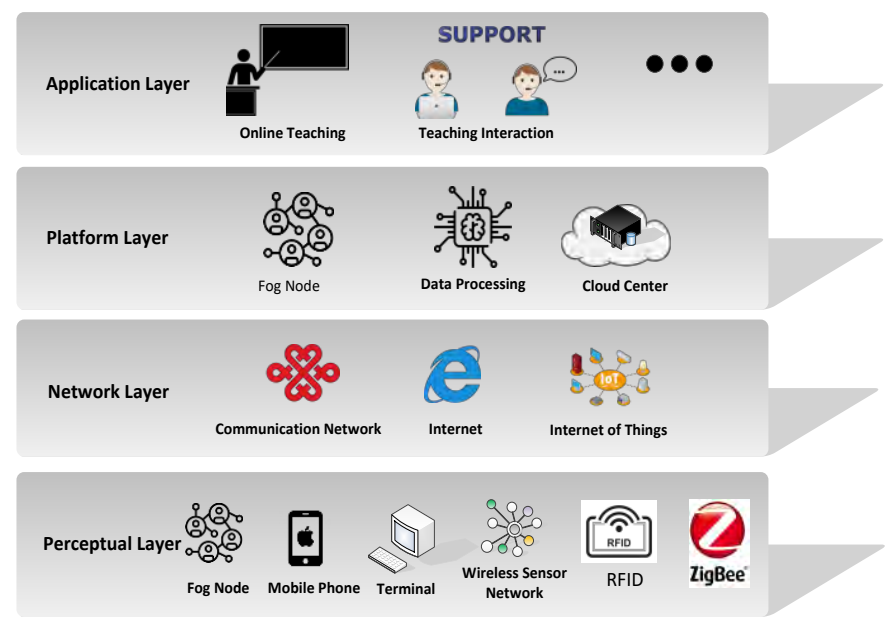

Fig. 1 Architecture of the innovative teaching platform

The Perception Layer, composed of a large number of terminals with sensing and communication functions and sensor networks and using sensor technology, RFID technology, ZigBee technology and so on to collect massive amounts of data which is transferred to the wireless network in a self-organizing manner, is the source of a large amount of computing data for the Internet of Things. Meanwhile, it deploys a large number of fog nodes, and the collected massive data is first uploaded to the nearest fog computing node for data processing and fusion, avoiding the network congestion caused by a large amount of data being simultaneously uploaded into the cloud.

The Network Layer is similar to the neural network of the human body, and transmit the data obtained from the Perception Layer securely, reliably and availably. It consists of the communication network, the Internet, and the Internet of Things. The communication network can be the mobile communication network used by different cities. The Internet refers to a network based on the Internet, cloud and fog, while the Internet of Things refers to a network based on M2M technology, which is a new generation of network between objects and objects.

The Platform Layer is the core part of the overall system, which includes a higher level of fog nodes, cloud servers, switches and so on. The network teaching platform intelligently processes the data transmitted from the previous layer, and transmits valuable information to the cloud through data analysis, processing and fusion, reducing the pressure on the cloud and facilitating global planning.
The Application Layer mainly analyzes the results after data processing and provides various solutions for the applications of the teaching platform. Its applications include online learning, teaching management, teaching resource sharing, teacher-student interaction, etc. ${ }^{[6]}$.

\section{2) Innovative teaching platform fog model}

In the fog model of the innovative teaching platform, it is assumed that each classroom is a fog node. Then, when students make a request such as enquiries or downloads in the process of online teaching, if the cloud computing model commonly used today is adopted, it is easy to cause large numbers of users to visit remote servers at the same time and in a short time, which not only leads to long communication distance and long delay, but also easily causes network congestion, influencing the experience of the teaching process. If the fog model in Fig.2 below is used, the user or the terminal directly communicates with the fog node close to the user to process the request, which not only greatly shortens the communication distance, but also reduces the delay. At the same time, The number of users processed synchronously by fog nodes which regard the classroom as the unit is relatively small, which is not easy to cause congestion. After the user requests are processed and fused in this layer, and the response is given, then they communicate with the fog node or the cloud at a higher level to relieve the pressure of data processing in the cloud. As for the fog nodes that are deployed close to the users, some of the required content can be cached in advance to serve nearby users or terminals more quickly and securely. As shown in Fig.2.

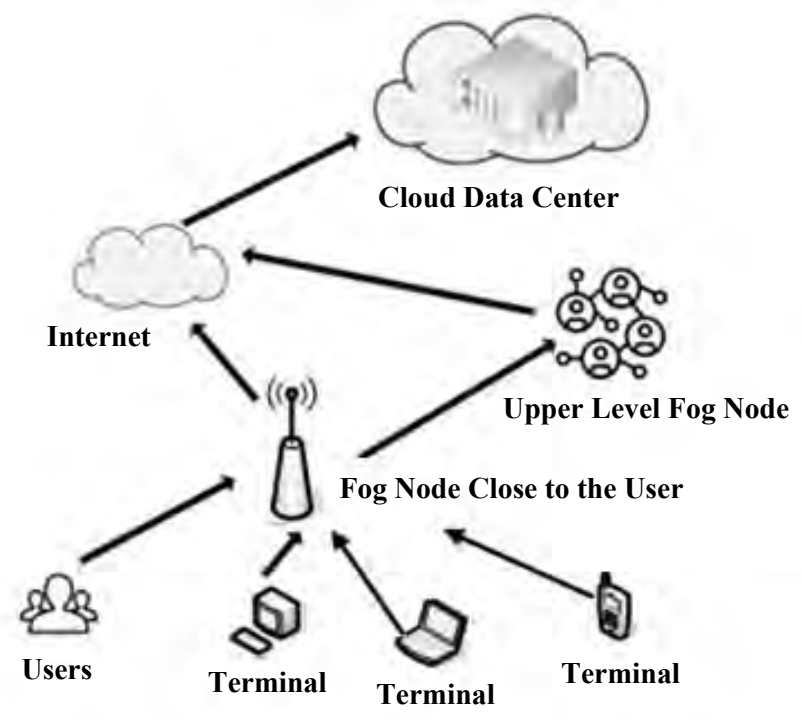

Fig. 2 Innovative teaching platform fog model 


\section{The role of fog computing in innovative teaching platforms}

\section{1) Reduce broadband pressure in online teaching platforms}

The innovative teaching platform based on fog computing distributes a large number of fog nodes close to the users. When the end users request query or download, first the nearby fog node is accessed instead of directly accessing the distant cloud as in the past, which is equivalent to insert the layer of fog computing into the cloud computing model. It not only shortens the communication distance, but also avoids the network congestion caused by massive users simultaneously accessing the cloud, reducing the broadband pressure in the network teaching process and improving the smoothness of online teaching.

\section{2) Reduce the burden on cloud servers and improve platform computing power}

Compared with cloud computing, fog computing has the advantage of "distributed computing", that is, the data processing function is no longer only concentrated in the cloud and each fog node has a data processing function, which makes it possible to preprocess the collected data. In the past, cloud computing uploaded all the collected data to the cloud and completed the data processing process in the cloud. Due to the current expansion of colleges and universities, not only the number of teaching classes and students has increased exponentially, but also the school district and the number of users' visits has increased rapidly, bringing great burden to the school cloud server and even causing its paralysis. The introduction of fog computing effect will make difference, because the fog computing calculates, processes, and fuses the collected data in the fog node first, and then transmits the processed data to the cloud, thus reducing the burden of the cloud server's processing and calculating redundant data, improving platform computing efficiency, and ensuring the smooth progress of all aspects of teaching.

\section{3) Reduce delay and enhance information exchange between teachers and students}

A large number of network fog nodes are located at the edge of the network, close to the user groups. The users are directly connected to the fog nodes without having to take a detour to connect to the cloud, thus shortening the communication distance. At the same time, the fog nodes have the function of processing data. The data can be processed directly in the nearby fog node instead of uploading to the cloud regardless of whether it is useful or not, which reduces the delay and facilitates the decision-making. In addition, the information exchange speed between teachers and students is also greatly improved, the real-time interaction effect between teachers and students is more convenient, and the huge role of new information technology in the innovation teaching platform can be truly experienced.

\section{APPLICATION OF FOG CALCULATION IN INNOVATIVE TEACHING PLATFORM}

\section{A. Information service}

The big data information service is very beneficial to both the school and the students. For the students, fog and all the teaching resources inside the school are assessable and students from different regions enjoy the same and superior teaching resources. To a certain extent, it reduces the unfairness of education. The platform can analyze students' learning records and recommend relevant teaching resources according to students' interests, plan their studying and provide efficient learning methods. The platform records the student's learning path and learning style, and uses big data analysis to predict the student's learning status and make teaching adjustments. When the school hosts events, such as lectures, competitions, and the school sports meeting, students can receive message sent by school on the innovative teaching platform, and students can participate in the latest activities in time. For the school, students can ask questions to the students through the platform, or send request to the classroom. The questions can be solved in time by the teachers or classmates, which improves the interaction between teachers and students and the interactivity between students and students. The students' learning situation can be aware in real time and clearly, and we can know which students may need the guidance of the school in time, so as to improve the students' innovative learning ability and quality. By analyzing the students' feedback on the course, the school can adjust the teaching plan accordingly. Besides, the school can quickly send notifications to each student through the innovative teaching platform. In short, the platform can provide information services to teachers and students accurately and in a timely manner.

\section{B. Distributed Computing}

The fog model has a large number of fog nodes. Although the calculation capability of each fog node device is relatively weak, the model uses a distributed computing scheme to effectively ensure that all fog nodes can play their roles, which can make full use of the computing power of the idle fog nodes, cut the huge task into several smaller tasks and they are assigned to each idle fog node, improving the calculation capability of the fog model, reducing the damage caused by the overload of individual fog nodes, and reducing the funds for school maintenance equipment. With distributed computing, setting up the environment-aware sensors in the classroom, and computing and analyzing the environmental information collected by the sensors, such as temperature, number of students and light can intelligently adjust classroom equipment and reduce resource waste. Distributed computing can analyze students' performance in the class to get the type of class and the teaching style that students like. Analyzing the students' search records with distributed computing can figure out what teaching resources are missing from the school, and feedback the results of the analysis to the school, and then the school can purposefully increase the teaching resources within the school. 


\section{Reduce storage pressure and release space at any time}

The data can be filtered, analyzed and processed by the fog nodes which filter a large amount of useless data and noise data through the function of preprocessing to prevent a large amount of useless data from entering the cloud, some data is stored in the fog node, and only a small amount of useful data can enter the cloud thus the storage pressure in the cloud is reduced. If the storage capacity of the fog nodes reaches a certain level, the fog nodes will delete the useless data autonomously according to the algorithm, and release the space at any time according to the storage requirement. The deleted data features that it has an earlier acquisition time, has been analyzed and processed, and does not affect the characteristics of the operation of the teaching platform.

\section{CONCLUSION}

Combined with fog computing technology, the innovative teaching platform reduce the pressure on cloud storage, computing and processing, reduce network broadband pressure, ensure low latency, enhance the interactive effect of teachers and students, and improve the sharing of teaching resources and students' autonomy in innovative learning. Teaching resources sharing makes the excellent teaching resources play a greater role. The interaction between teachers and students enhances the friendship between teachers and students. Not only can it promptly answer questions for students, but also can improve students' innovative ability training and knowledge level. What is more important is that students can learn independently through innovative teaching platforms, regardless of time and place. Students are not limited by time and space, maximizing the innovative learning environment and improving the quality of teaching.

\section{REFERENCES}

[1] Qu Hongyi, Han Xibin, Zhang Ming et al. Research progress of network teaching platform [J]. China Distance Education, 2006 (5S): 55-59. (In Chinese)

[2] Cao Wenzhuo. Research on Application of Learning Analysis Based on Cloud Classroom Learning Platform [D]. Central China Normal University, 2016. (In Chinese)

[3] Han Yun. Design and implementation of network teaching platform based on three-tier architecture [D]. Ocean University of China, 2010. (In Chinese)

[4] Bonomi F,Milito R,zhu J,et al.Fog Computing and its Role in the Internet of Things.Edition of the Mcc Workshop on Mobile Cloud Computing. ACM, 2012:13-16.

[5] Yu Shengquan, He Kekang. Architecture and Function of Network Teaching Platform [J]. China Electro-education Education, 2001 (08): 60-63. (In Chinese)

[6] Chongyang. Design of network teaching platform based on cloud computing [J]. Electronic Testing, 2016 (06): 69-70. (In Chinese) 